\title{
Medical uses for phenol in the urinary tract: A possible forgotten treatment (Review)
}

\author{
TAKUYA SADAHIRA ${ }^{1}$, YUKI MARUYAMA ${ }^{1}$, TOYOHIKO WATANABE $^{1}$, TAKANORI SEKITO $^{1}$, \\ YOSUKE MITSUI $^{1}$, KOICHIRO WADA ${ }^{2}$, MOTOO ARAKI $^{1}$ and MASAMI WATANABE ${ }^{3}$ \\ ${ }^{1}$ Department of Urology, Okayama University Graduate School of Medicine, Dentistry and Pharmaceutical Sciences, \\ Okayama 700-8558; ${ }^{2}$ Department of Urology, Shimane University School of Medicine, Izumo, Shimane 693-0021; \\ ${ }^{3}$ Center for Innovative Clinical Medicine, Okayama University Hospital, Okayama 700-8558, Japan
}

Received July 15, 2021; Accepted August 30, 2021

DOI: $10.3892 / \mathrm{mi} .2021 .13$

\begin{abstract}
Phenol is a chemical compound that was first used medically as an antiseptic. At low concentrations, phenol exerts local anesthetic effects achieved through denervation; at high concentrations, it exerts a potent protein-denaturing effect that induces apoptosis. Phenol injection therapy has a long history of use in urology. It is reportedly effective for hemorrhagic cystitis, benign prostate hyperplasia, overactive bladder, hydrocele, bladder tumors, interstitial cystitis and other benign urologic diseases, and it is also used as a tool to decrease bleeding during prostate surgery. The present review article summarizes the medical applications of phenol in urological field. The articles available on the medical uses of phenol are primarily older and retrospective, involving small numbers of patients. In the absence of comparative studies with other treatments, it is impossible to determine the relative benefit of phenol. However, the treatment outcomes of phenol injection are fairly well-established. Phenol therapy may be an option for patients who are poor candidates for invasive treatment. Further studies are required, however, as are improvements in the injection technique to reduce the rate of complications.
\end{abstract}

\section{Contents}

1. Introduction

2. Medical uses for phenol in the bladder

3. Medical uses for phenol in the prostate

4. Uses of phenol in other diseases

5. Conclusion and future perspectives

Correspondence to: Professor Masami Watanabe, Center for Innovative Clinical Medicine, Okayama University Hospital, 2-5-1 Shikata-cho, Okayama 700-8558, Japan

E-mail:masami5@md.okayama-u.ac.jp

Key words: phenol, urinary tract, urinary bladder, therapeutic

\section{Introduction}

Joseph Lister first used phenol in a medical capacity in 1865 , as an antiseptic to sterilize operating fields, with favorable results (1). In addition to its antimicrobial actions, phenol exerts local anesthetic effects at low concentrations. These effects are achieved through denervation $(2,3)$. At high concentrations, phenol exerts a potent protein-denaturing effect (4). Due to its various effects, phenol is used in a wide range of fields (3-9). However, there are only a limited number of reviews available on the medical applications of phenol. In the present review, the medical applications of phenol in urological field are summarized.

\section{Medical uses for phenol in the bladder}

Hemorrhagic cystitis (HC). $\mathrm{HC}$ is a condition associated with certain disease states, as well as with exposure to drugs, viruses and toxins. Diffuse inflammation causes bleeding from the bladder mucosa (9). Management options include coagulation, embolization, fulguration, hyperbaric oxygen therapy and surgical treatment (10). However, some patients experience intractable $\mathrm{HC}$ that is difficult to manage, particularly when the condition develops after the use of cyclophosphamide chemotherapy. In such patients, the intravesical instillation of phenol is an effective, minimally invasive and less morbid option compared with conventional treatments (11). The intravesical instillation of phenol is reportedly effective for chemical cauterization in patients with severe bladder hemorrhage $(11,12)$. In a clinical experiment, phenol was shown to destroy the urothelium, but not muscle (13). The bladder capacity diminishes temporarily and then returns to its normal size with the complete epithelialization of the urothelium. The treatment is associated with a low incidence of bladder fibrosis (13). A reported complication of phenol involved failure to drain the bladder following instillation. The infant suffered severe methemoglobinemia (14).

Previous case reports describe two children with leukemia, 12 and 16 years of age, who were administered phenol for refractory HC following cyclophosphamide therapy $(14,15)$. The method requires suprapubic cystostomy under anesthesia. A total of $30 \mathrm{cc}$ of $100 \%$ phenol with $30 \mathrm{cc}$ of glycerin is 
instilled and left in the bladder for $1 \mathrm{~min}$. The mixture is then removed by suction followed by the instillation of $60 \mathrm{cc}$ absolute ethanol (90 or 95\%) to neutralize the effect of any remaining phenol. After $1 \mathrm{~min}$, the ethanol is suctioned out and the bladder is irrigated with copious amounts of saline. The bladder is closed, and a $30 \mathrm{~F}$ suprapubic Foley catheter is left in place $(14,15)$. Both case reports describe the immediate post-operative clearing of the urine, and both patients had their suprapubic catheter removed 6 weeks after surgery $(14,15)$. Initially, the patients had a small bladder capacity on intravenous pyelogram and were unable to feel the sensation of needing to void. Within 2-4 weeks, the patients recovered sensation, reasonable bladder control and adequate bladder capacity. Both of these reports describe the use of ethanol to wash out the remaining phenol in the bladder $(14,15)$. Phenol does not dissolve in water at relatively high concentrations, although it does dissolve uniformly in water at low concentrations $(\leq 7 \%)(16)$. Thus, it may be possible to omit the step of ethanol instillation.

Phenol is also effective when silver nitrate fails to control bleeding (17). The mucosa is swabbed with a 1:1 phenol-alcohol solution, and bleeding is immediately terminated. Duckett et al (15) described the successful control of $\mathrm{HC}$ by phenol cauterization in three patients, although the details of treatment are unclear.

Severe vesical bleeding in children is difficult to manage as the small urethra precludes the use of large catheters for bladder irrigation and clot removal. Thus, phenol may be particularly useful in pediatric patients (18). However, since experience with phenol is limited, further investigations are required to determine the usefulness of injection therapy.

Overactive bladder. Subtrigonal phenol injection has been employed since 1982 for patients with detrusor instability, detrusor hyperreflexia and bladder hypersensitivity who have not had success with more conservative treatments (19). Ewing et al (19) were the first to describe this technique in 1982. Cystoscopy is performed under general anesthesia, and a 35-cm, 20-gauge Shuttleworth needle is passed through the bladder wall and advanced to midway between the bladder neck and the ureteric office on each side. A total of $10 \mathrm{ml} 6 \%$ phenol solution is injected into each side through transvaginal approach. An indwelling Foley catheter is left overnight and removed the following morning. The overall response rate in the 30 patients in the study by Ewing et al (19) was $62.5 \%$ at 1 year. In subsequent studies, the results of treatment in the early post-injection period ranged from 29 to $63 \%$ (20-25). Blackford et al reported the highest number of patients: 62 of their 116 patients $(53 \%)$ had a symptomatic response at 3 months after the phenol injection (25). A satisfactory response rate was more likely in patients with detrusor hyperreflexia $(82 \%)$, older patients with detrusor instability $(69 \%)$ and patients with idiopathic bladder hypersensitivity $(68 \%)$, but less likely in younger patients with detrusor instability (14\%) and in patients who had bladder hypersensitivity with a definable cause, such as interstitial cystitis (0\%) (25). Other researchers have noted a diminishing effect of infusion therapy over time. Rosenbaum et al (23) reported that the overall success rate at 1 month was $48 \%$, decreasing to $16 \%$ at 6 months and to $3 \%$ at 1 year. Chapple et al (21) also reported that 10 of their 18 female patients exhibited an improvement in their condition at 1 month; however, this figure decreased to 7 out of 18 at 3 months and to 2 out of 18 at 6 months. These results demonstrate that clinical denervation following a transtrigonal phenol injection with a $6 \%$ phenol aqueous solution may be, at best, only transient.

Some concerning complications are sometimes observed after the injection. Ewing et al (19) reported that 5 of their 30 patients experienced chronic urinary retention, and 2 patients experienced a trigonal ulcer and transient ureteric reflux; the ulcer and the reflux resolved spontaneously. Mclnerney et al (20) also described a significant complication rate $(17 \%)$ for phenol injection. The most common complication in their study was urinary retention, occurring in 8 of 72 patients (20). In addition, 4 patients experienced nerve palsies, and 2 patients developed localized bladder mucosal necrosis at the injection site; one of these patients experienced calculus formation. One of the 9 male patients developed erectile dysfunction (20). The authors of other series have reported fistula formation, significant hematuria and ureteric stenosis (21-25). Vesicovaginal fistula is a particularly severe complication that can arise from placing phenol too medially. Extreme caution should be exercised when a second injection is necessary, e.g., in patients with an inadequate response to an initial injection.

These findings suggest that the use of transtrigonal phenol for the treatment of overactive bladder is difficult to justify using the reported methods. One reason for the poor results and the resultant complications may be that phenol diffuses widely through the perivesical fat (23). Improving the injection technique to prevent phenol diffusion could lead to greater efficacy and may prevent complications. Further research and development in this area is required.

Bladder tumors. Multiple papillomatosis of the bladder and diffuse non-infiltrating papillary carcinoma of the bladder are conditions that are difficult to assess using transurethral techniques. Unless each individual lesion is removed and staged, the variation in malignant potential among lesions cannot be accurately assessed. Vermooten et al (26) overcame this difficulty by using phenol to induce complete destruction of the bladder mucosa in the hope that the newly regenerated epithelium would not show a neoplastic tendency. Modifying a suggestion of Kirwin (27), they reported the successful use of phenol for chemical cauterization of bladder mucosal tumors in 13 patients with papillomatosis (26), using a solution consisting of equal parts pure phenol and glycerin. The bladder capacity was initially diminished but later returned to an adequate size. Treatment appears to be efficient in ridding the bladder mucosa of papillary lesions, and recurrences are infrequent following the complete destruction of the bladder mucosa, though they do occasionally occur. Filling the bladder with phenol does not appear to be associated with treatment-related mortality.

To investigate how deeply phenol penetrates and the time required for regeneration of the bladder epithelium, Vermooten et al (26) performed experiments using canines. Under general anesthesia, the bladder was filled with $150 \mathrm{cc}$ of a solution of equal parts pure phenol and glycerin; the solution was left in place for $2 \mathrm{~min}$. The mixture was then removed and the bladder was washed with $95 \%$ alcohol. The animals 
were serially sacrificed. At 5 days, the intravesical ureter and ureteral orifice were noted to have an intact epithelium with a fibropurulent eschar replacing the destroyed bladder epithelium. Edema and infiltration did not extend into the muscular layer. At 7 days, the epithelium had begun to regenerate from the ureteral orifices, replacing the eschar on the mucosal surface. At 21 days, the bladder mucosal epithelium had regenerated to its normal thickness, inflammation and edema had diminished, and there was no evidence of dysplasia or neoplasia. Cystography revealed no evidence of reflux. Phenol injection for chemical cauterization of the bladder mucosa for papillomatosis appears to be safe, based on this animal model (26).

Interstitial cystitis. Interstitial cystitis is a disease of unknown etiology in which the main symptoms are bladder pain, urinary frequency and urgency (28). Particularly in the presence of Hunner's ulcers, it is associated with severe, recurrent bladder pain. The glycosaminoglycan layer of the bladder mucosa is impaired in patients with interstitial cystitis, and sensory nerves, such as the unmyelinated $\mathrm{C}$-fibers are stimulated by urine, causing pain. A number of types of treatment have been advocated $(29,30)$, including denervation using local application of phenol in the bladder. Investigators have applied various strengths of phenol solution directly to the ulcer $(31,32)$; the use of $50 \%$ phenol and pure phenol are reportedly effective (29). The application of phenol causes protein coagulation, which leads to non-selective tissue destruction and the initiation of degeneration in nerve fibers, with a neurolytic effect that lasts for several months (32). However, neurodestructive modalities, including phenol, have potential complication and adverse effects. Advances in phenol injection techniques are required to solve these issues. The authors of the present review previously developed a novel, in situ permeation system that controlled the intratissue diffusion of therapeutic agents (33). This system will enable the localized ablation of target lesions.

\section{Medical uses for phenol in the prostate}

Injection into the prostate. Phenol injection has also been used in the treatment of diseases of the prostate (34-36). The origin of intraprostatic phenol injection therapy was described by Talwar and Pande (37) in 1966, who treated 188 patients with benign prostate hyperplasia (BPH) with a $78.2 \%$ success rate. They used a composition of $2 \%$ liquid phenol, $2 \%$ glacial acetic acid, and $4 \%$ glycerin in distilled water. The patient was placed in the left lateral position and the prostate was injected with 2-3 $\mathrm{ml}$ phenol solution through a lumbar puncture needle. The needle was inserted into the perineum and guided into the gland using a finger in the rectum. An indwelling catheter was left in place and removed within 5-7 days. The injection was repeated if micturition was not restored in patients with urinary retention. If the $\mathrm{BPH}$ of the patient is of an early stage and the patient is not suffering from urinary retention, the injection can be performed as an outpatient procedure (37). Following this first description, there have been several reports of phenol injection for BPH in patients with or without urinary retention (34,38-40). The success rate varies from $56.4 \%$, reported by Sharma and Goel (34), to $100 \%$, reported by Shipman and Akile (39).
Intraprostatic phenol injection therapy is based on the concept of chemical necrosis and the consequent sloughing or shrinkage of obstructive tissue. In a study on 13 patients who received injection therapy, the mean estimated prostate size decreased from $31 \mathrm{~g}$ pre-operatively to $21 \mathrm{~g}$ post-operatively, and the residual urine volume was significantly improved (41). Complications are relatively rare and mild; these include hemorrhage, pain, impotence and poor urinary control. Perineal pain is the most common complication, occurring within a few minutes of injection and lasting up to several days $(38,41)$. Plain pelvic radiographs obtained after the injection indicate that perineal pain may be associated with the extravasation of the solution outside the prostatic capsule (41).

This technique may be particularly useful for select patients who are at risk of poor outcomes following surgical treatment. However, the exact efficacy of injection therapy remains unclear as it is not uncommon to observed the spontaneous restoration of the ability to void following a period of catheter drainage of the bladder, and symptoms may improve following placebo treatment $(42,43)$.

The intraprostatic injection of phenol solution has been adapted to minimize blood loss during prostatectomy or the transurethral resection of the prostate (TURP) $(35,36)$. In open radical prostatectomy, a transperineal injection of $2 \mathrm{ml}$ of $5 \%$ phenol in an almond oil solution is injected into the prostate at $48 \mathrm{~h}$ prior to surgery. A prospective study on 100 patients undergoing prostatectomy found that none of the 50 patients who received a phenol injection experienced perineal pain (35). In these patients, blood loss ranged from 20-120 ml, while blood loss in the non-injected group ranged from $175-680 \mathrm{ml}$. None of the injected patients required blood transfusion post-operatively, whereas six of the non-injected patients required transfusion for excessive blood loss or low hemoglobin values. Coagulative necrosis produced by the sclerosant phenol solution seems to reduce vascularity and renders it technically easier to enucleate the prostate, thereby reducing operative time (35).

In 1991, Szewczyk (36) used a 5\% phenol solution in almond oil for patients undergoing TURP. The volume of resected prostate tissue, operative time and blood loss were compared among three groups: Those who were administered an injection of phenol solution 2 days prior to TURP, those who were administered the injection 5 days prior to TURP and a non-injected group. No differences were observed between the groups for any of the three factors (36). It is possible that 5 days is an inadequate time frame to produce the necessary necrosis of prostatic tissue to diminish surgical bleeding. Currently, laparoscopic surgery, robotic surgery and holmium laser nucleation are the mainstays of treatment for prostate disease; the utility of phenol injection for these procedures is unclear.

Complications due to phenol injection are pain towards the tip of the penis, a temperature $>38^{\circ} \mathrm{C}$ persisting $<48 \mathrm{~h}$ after the injection, pain in the perineum and hematuria. There have never been any severe consequences or systemic side-effects reported due to phenol injection (35-43), at least to the best of our knowledge. However, cases of latent and occult prostate cancer may have not been noted and the injection may have increased the chance of metastases. In these studies, the injection was administered when prostate cancer had been excluded clinically. 


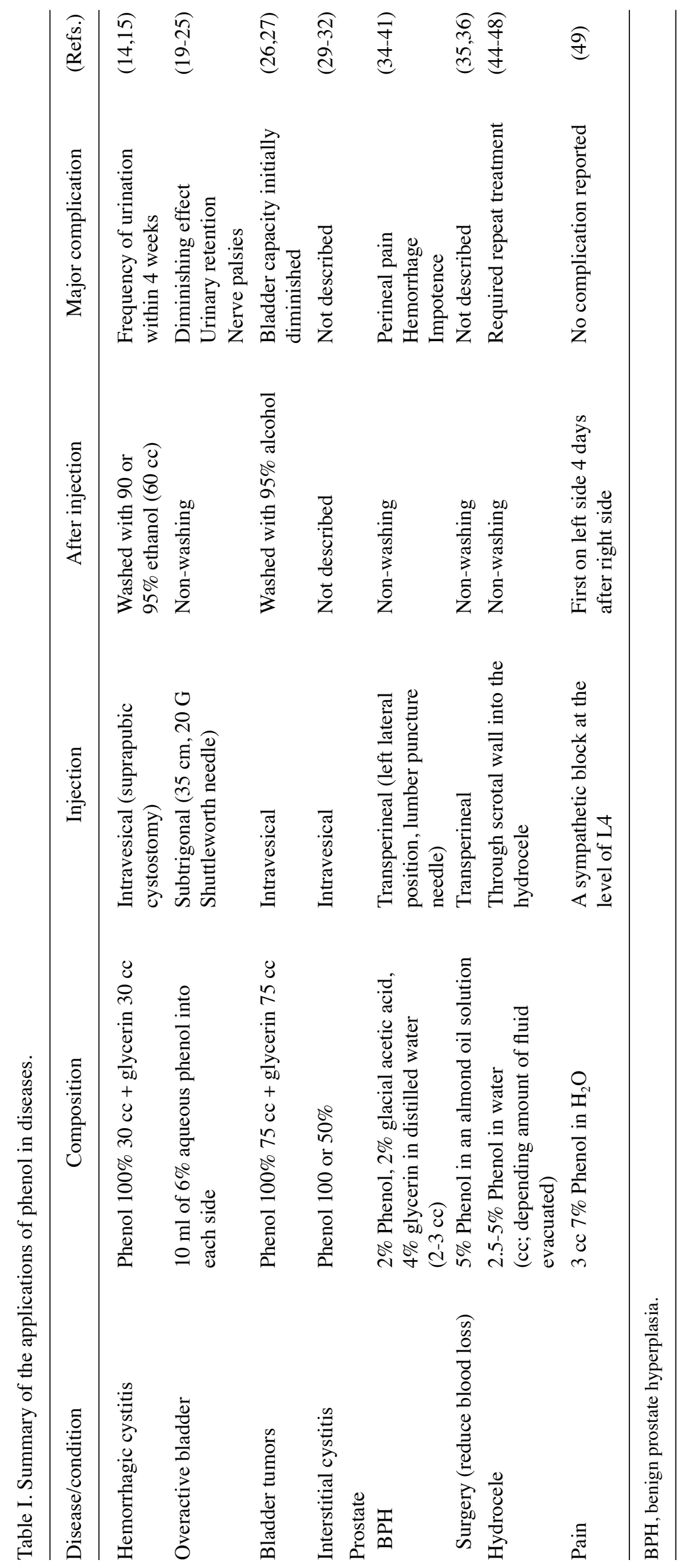




\section{Uses of phenol in other diseases}

Hydrocele. Sclerotherapy using phenol has been proposed as an alternative therapy to surgical treatment for patients with hydrocele $(44,45)$. Moloney (44) first reported the use of injection therapy in 1975 , using a $21 \%$ phenol solution. The treatment requires one to three outpatient visits, has an almost negligible complication rate and has had no treatment failures. Other investigators have used low concentrations of phenol: The cure rate after a single injection was reportedly 48 to $52 \%$ using $2.5 \%$ phenol, $58 \%$ using $3 \%$ phenol and $70.5 \%$ using $5 \%$ phenol (45-47). East and DuQuesnay (46) suggested that $5 \%$ phenol yielded significantly superior results than a $2.5 \%$ phenol injection $(\mathrm{P}=0.002$ and $\mathrm{P}=0.012)$, and a $100 \%$ cure rate was achieved following three to four instillations. As sclerotherapy has fewer complications, a lower morbidity and is more cost-effective than other treatments, a number of chemicals in addition to phenol have been investigated, including tetracycline, sodium tetradecyl sulfate, polidocanol, fibrin glue, picibanil (OK-432), ethanolamine oleate, antazoline, rifampicin and talc (48). Although the use of any of these treatments by injection may readily be performed in a consulting room, repeat treatment is sometimes required, the efficacy compared with surgery is controversial, and patient satisfaction is lower than that with surgery. Thus, injection therapy is not currently considered a standard treatment for hydrocele.

Sympathetic block for pain. The local anesthetic effects that phenol achieves through denervation have been applied to the use of sympathetic block for chronic prostatic pain. Patients with chronic prostatitis in whom conventional chemotherapy and repeated transurethral resection have failed to relieve pain are able to achieve complete relief with a permanent sympathetic block using phenol (49). Pain from the prostate gland can be mediated either via parasympathetic fibers from the caudal portion of the pelvic plexus or via sympathetic chain fibers from the lumbar segments of the spinal cord. Chemical sympathectomy using phenol (3 $\mathrm{ml} 7 \%$ phenol in water) is performed at L4, first on the left side and 4 days later on the right side. The needle position is carefully examined radiologically using both anteroposterior and lateral views. Complete pain relief is achieved without complication (49).

\section{Conclusion and future perspectives}

The therapeutic application of phenol is reportedly effective for a wide variety of urologic diseases (Table I). However, reports describing this therapy are primarily older and rely on small cohorts of patients. Currently, this treatment is not listed in the guidelines due to its complications. In additions, phenol, which is an environmental pollutant, and can cause acute renal failure, may require a more impartial and more careful interpretation and analysis. By changing the method of administration (e.g., local ablation), it is considered that highly effective drugs, such as phenol, which have been considered as deleterious drugs, can be used safely and may become a treatment option for refractory urinary tract diseases. Medical technologies are advancing daily, with techniques such as robotic surgery and laser therapy coming to the fore. The interest in minimally invasive treatment is increasing, and phenol offers the advantages of avoiding unnecessary hospitalization and surgery. However, in order for phenol to be used more effectively with fewer complications, it is necessary to improve the therapeutic techniques. In the future, phenol may play a role in the treatment of urinary tract diseases as an active medical agent.

\section{Acknowledgements}

Not applicable.

\section{Funding}

No funding was received.

\section{Availability of data and materials}

The datasets used and/or analyzed during the current study are available from the corresponding author on reasonable request.

\section{Authors' contributions}

TSa wrote the manuscript. TSa, YMa, TW, TSe and MW made substantial contributions to the conception and design of the present study and involved in the literature search. TW, YMi, KW and MA made substantial contributions to and were involved in the literature search and study selection for the review. TSa, YMa, KW and MW made substantial contributions to the conception and design of the study, provided critical points of discussion and were involved in the completion of the manuscript. TSa and MW confirm the authenticity of all the raw data. All authors have read and approved the final manuscript.

\section{Ethics approval and consent to participate}

Not applicable.

\section{Patients consent for publication}

Not applicable.

\section{Competing interests}

The authors declare that they have no competing interests.

\section{References}

1. Downs JW and Wills BK: Phenol toxicity. In: StatPearls [Internet]. StatPearls Publishing, Treasure Island, FL, 2021.

2. Robertson A, Whitwell $\mathrm{R}$ and Osborne J: Is phenol a safe local anaesthetic for grommet insertion? J Laryngol Otol 120: 20-23, 2006.

3. Sing T: Use of phenol in anaesthetizing the eardrum. The Internet Journal of Otorhinolaryngology 4: 1-4, 2019.

4. Lack W, Lang S and Brand G: Necrotizing effect of phenol on normal tissues and on tumors. A study on postoperative and cadaver specimens. Acta Orthop Scand 65: 351-354, 1994.

5. Costa A, Bonner MY and Arbiser JL: Use of polyphenolic compounds in dermatologic oncology. Am J Clin Dermatol 17: 369-385, 2016.

6. Sasagawa T, Fujita S, Osaka Y, Sakamoto J, Shibata T, Oka Y, Tomizawa H, Takakura M and Takagi H: A novel ablation therapy for cervical and vaginal squamous intraepithelial lesions using a liquid phenol. J Kanazawa Med Univ 42: 63-68, 2017 (In Japanese) 
7. Botte MJ, Abrams RA and Bodine-Fowler SC: Treatment of acquired muscle spasticity using phenol peripheral nerve blocks. Orthopedics 18: 151-159, 1995.

8. Yano $\mathrm{T}$ and Yano K: Comparison of injection sclerotherapy between $5 \%$ phenol in almond oil and aluminum potassium sulfate and tannic acid for grade 3 hemorrhoids. Ann Coloproctol 31: 103-105, 2015.

9. Manikandan R, Kumar S and Dorairajan LN: Hemorrhagic cystitis: A challenge to the urologist. Indian J Urol 26: 159-166, 2010.

10. Nabi G, Sheikh N, Greene D and Marsh R: Therapeutic transcatheter arterial embolization in the management of intractable haemorrhage from pelvic urological malignancies: Preliminary experience and long-term follow-up. BJU Int 92: 245-247, 2003.

11. deVries CR and Freiha FS: Hemorrhagic cystitis: A review. J Urol 143: 1-9, 1990.

12. West NJ: Prevention and treatment of hemorrhagic cystitis Pharmacotherapy 17: 696-706, 1997.

13. Susan LP and Marsh RJ: Phenolization of bladder in treatment of massive intractable hematuria. Urology 5: 119-121, 1975.

14. Lebowitz RL: Intravesical chemical cauterization and methemoglobinemia. Pediatrics 65: 630, 1980.

15. Duckett JW Jr, Peters PC and Donaldson MH: Severe cyclophosphamide hemorrhagic cystitis controlled with phenol. J Pediatr Surg 8: 55-57, 1973

16. Campbell AN and Campbell JR: Concentrations, total and partial vapor pressures, surface tensions and viscosities, in the systems phenol-water and phenol-water-4\% succinic acid. J Am Chem Soc 59: 2481-2488, 1937.

17. Kumar AP, Wrenn EL Jr, Jayalakshmamma B, Conrad L, Quinn P and Cox C: Silver nitrate irrigation to control bladder hemorrhage in children receiving cancer therapy. J Urol 16 85-86, 1976

18. Redman JF and Kletzel M: Cutaneous vesicostomy with direct intravesical application of formalin: Management of severe vesical hemorrhage resulting from high dose cyclophosphamide in boys. J Urol 151: 1048-1050, 1994.

19. Ewing R, Bultitude MI and Shuttleworth KE: Subtrigonal phenol injection for urge incontinence secondary to detrusor instability in females. Br J Urol 54: 689-692, 1982

20. Mclnerney PD, Vanner TF, Matenhelia S and Stephenson TP Assessment of the long-term results of subtrigonal phenolisation. Br J Urol 67: 586-587, 1991.

21. Chapple CR, Hampson SJ, Turner-Warwick RT and Worth PH: Subtrigonal phenol injection. How safe and effective is it? $\mathrm{Br}$ J Urol 68: 483-486, 1991.

22. Wall LL and Stanton SL: Transvesical phenol injection of pelvic nerve plexuses in females with refractory urge incontinence. Br J Urol 63: 465-468, 1989.

23. Rosenbaum TP, Shaw PJ and Worth PH: Trans-trigonal phenol failed the test of time. Br J Urol 66: 164-169, 1990.

24. Cameron-Strange A and Millard RJ: Management of refractory detrusor instability by transvesical phenol injection. Br J Urol 62 : 323-325, 1988

25. Blackford HN, Murray K, Stephenson TP and Mundy AR: Results of transvesical infiltration of the pelvic plexuses with phenol in 116 patients. Br J Urol 56: 647-649, 1984.

26. Vermooten V, Peter PC and Johnson DE: Treatment of papillomatosis of the bladder with phenol and glycerin: A clinical and laboratory study. J Urol 99: 588-592, 1968.

27. Kirwin TJ: Tumors of the bladder. J Internat Coll Surg 13: 1-19, 1950.

28. Akiyama Y, Luo Y, Hanno PM, Maeda D and Homma Y: Interstitial cystitis/bladder pain syndrome: The evolving landscape, animal models and future perspectives. Int J Urol 27 $491-503,2020$
29. William J and Engel MD: Interstitial Cystitis. Cleve Clin J Med 6: 307-312, 1939

30. Sears NP: Elusive ulcer of the bladder; with special reference to its treatment with phenol. New York State J Med. 1936; 36: 724-728, 1936.

31. Thomas LP and Hugh FR: Interstitial cystitis: Treatment with silver nitrate. J Urol 51: 520-525, 1944.

32. Kim JH, Kim E and Kim BI: Pulsed radiofrequency treatment of the superior hypogastric plexus in an interstitial cystitis patient with chronic pain and symptoms refractory to oral and intravesical medications and bladder hydrodistension: A case report. Medicine (Baltimore) 95: e5549, 2016.

33. Watanabe M: A novel in situ permeation system and its utility in cancer tissue ablation. Int J Oncol 47: 875-883, 2015.

34. Sharma GD and Goel PP: Transperineal intraprostatic injection treatment of benign prostatic enlargement. Aust N Z J Surg 47: 220-222, 1977

35. Muzafer MH: Blood loss in prostatectomy. Comparison between injected and non-injected prostates. Int Urol Nephrol 18: 163-167, 1986.

36. Szewczyk W: Blood loss during transurethral resection of the prostate injected with phenol solution. Int Urol Nephrol 23: $155-159,1991$.

37. Talwar GL and Pande SK: Injection treatment of enlarged prostate. Br J Surg 53: 421-427, 1966.

38. Angell JC: Treatment of benign prostatic hyperplasia by phenol injection. Br J Urol 41: 735-738, 1969.

39. Shipman JJ and Akilie AN: Treatment by prostatic injection of acute urinary retention due to prostatic hyperplasia. Br Med J 2: 418-419, 1967.

40. Choudhury A and Maulik AK: Evaluation of the role of injection therapy for benign prostatic hypertrophy. Br J Urol 2: 204-207, 1980.

41. Broughton AC and Smith PH: The significance of perineal pain after injection of the prostate. Br J Urol 42: 73-75, 1970.

42. Castro JE and Griffiths HL: The assessment of patients with benign prostatic hypertrophy. J R Coll Surg Edinb 17: 190-194, 1972.

43. Laufer WE: Phenol injection for benign prostatic hypertrophy. Trop Doct 28: 98-99, 1998.

44. Moloney GE: Comparison of results of treatment of hydrocele and epididymal cysts by surgery and injection. Br Med J 3: 478-479, 1975.

45. Shan CJ, Lucon AM and Arap S: Comparative study of sclerotherapy with phenol and surgical treatment for hydrocele. J Urol 169: 1056-1059, 2003.

46. East JM and DuQuesnay D: Sclerotherapy of idiopathic hydroceles and epididymal cysts: A historical comparison trial of 5\% phenol versus tetracycline. West Indian Med J 56: 520-525, 2007.

47. Savion M, Wolloch Y and Savir A: Phenol sclerotherapy for hydrocele: A study in 55 patients. J Urol 142: 1500-1501, 1989.

48. Khaniya S, Agrawal CS, Koirala R, Regmi R and Adhikary S: Comparison of aspiration-sclerotherapy with hydrocelectomy in the management of hydrocele: A prospective randomized study. Int J Surg 7: 392-395, 2009.

49. Johansson $\mathrm{H}$ : Chemical sympathectomy with phenol for chronic prostatic pain. Eur Urol 2: 98-99, 1976.

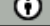

This work is licensed under a Creative Common Attribution 4.0 International (CC BY 4.0) License. 